\title{
Ansätze für eine Neue Normalarbeitszeit Ein Diskussionsbeitrag
}

\author{
Johannes GÄRTNER ${ }^{\mathrm{ab}}$, Anna ARLINGHAUS ${ }^{\mathrm{a}}$, Peter BAUMGARTNER ${ }^{\mathrm{a}}$, \\ Karin BOONSTRA-HÖRWEIN ${ }^{\mathrm{a}}$, Werner MARSCHITZa, Ruth SIGLÄR ${ }^{\mathrm{a}}$
}

${ }^{a}$ XIMES GmbH, Wien, Österreich; ${ }^{b} T U$ Wien, Wien, Österreich

\begin{abstract}
Die aktuelle Regulierung von Arbeitszeiten schafft Anreize für belastende Zeiten und berücksichtigt nur unzureichend wissenschaftliche Erkenntnisse zu den Auswirkungen dieser Belastungen. Dieser Beitrag stellt einen neuen Ansatz vor: Ausgehend von einer einerseits weit verbreiteten und andererseits auch sozialund gesundheitspolitisch wünschenswerten ,Neuen Normalarbeitszeit', nämlich der Gleitzeit von Montag bis Freitag tagsüber, sollen abweichende Arbeitszeiten hinsichtlich ihrer Belastung bewertet werden. Eine angemessene Kompensation in zusätzlicher Freizeit statt finanzieller Zulagen soll dann diese Belastung ausgleichen statt sie finanziell abzugelten. Damit würden auch bestehende Anreize für ein freiwilliges Verbleiben in belastenden Arbeitszeiten reduziert.
\end{abstract}

Keywords: Arbeitszeit, Gleitzeit, Belastung, Arbeitsrecht

\section{Einleitung}

Die österreichischen Regulative im Bereich der Arbeitszeit weisen - mit Ausnahme der Novelle 2018 - viele positive Eigenschaften auf, insbesondere die konsensorientierten, an den jeweiligen Rahmenbedingungen ausgerichteten Möglichkeiten der mehr oder weniger starken Beschränkung bestimmter Zeitdauern und Mengen.

$\mathrm{Zu}$ den ungünstigen Eigenschaften zählt, dass Entgeltfragen sehr stark mit Fragen der Zeitgestaltung (Risiken, Gesundheit, Soziales) verwoben sind und zum Teil die Erzielung höherer Einkommen von den Sozialpartnern, aber auch von Individuen, höher ge-

\footnotetext{
${ }^{1}$ Dr. Gärtner ist Gründer und Geschäftsführer der XIMES GmbH. Neben seinen Beratungstätigkeiten bei der XIMES GmbH unterrichtet Dr. Gärtner an der Technischen Universität Wien und ist im Vorstand der Arbeitszeitgesellschaft. Kontakt: Dr. Johannes Gärtner, XIMES GmbH, Hollandstraße 12, A-1020 Wien, Österreich. E-Mail: gaertner@ximes.com. Das AutorInnen-Team setzt sich aus Senior Consultants der XIMES GmbH zusammen, deren Arbeitsschwerpunkte rund um die Gestaltung von Arbeitszeit, Analyse und Berechnung von Personalbedarf sowie Entgelt liegen: Dr. Anna Arlinghaus., E-Mail: arlinghaus@ximes.com; Mag. Peter Baumgartner, E-Mail: baumgartner@ximes.com; Dr. Karin Boonstra-Hörwein, E-Mail: hoerwein@ximes.com; Mag. Werner Marschitz, E-Mail: marschitz@ximes.com; Mag. Ruth Siglär, E-Mail: siglaer@ximes.com.
} 
wertet wird als andere Faktoren (zum Beispiel manchmal zu Lasten der Allgemeinheit bei unfallträchtiger Arbeit). So gerät die Frage der optimalen Zeitgestaltung (etwa bei betrieblichen Flexibilitätswünschen) im Vergleich zum Entgeltthema oft in den Hintergrund. Ein Beispiel: Niedrige Überstundenzuschläge (oder einfach anzuordnende Überstunden) machen Überstunden für Unternehmen attraktiv; hohe Überstundenzuschläge führen aber wiederum dazu, dass Beschäftigte - vor allem nach Monaten der Gewöhnung - darum kämpfen, diese auch langfristig zu behalten (siehe auch Gärtner et al. 2014) - auch unter Nichtbeachtung der eigenen kurz- und langfristigen Gesundheit sowie Work-Life-Balance.

Ein weiterer Nachteil der derzeitigen Regulierung ist, dass es nur eine lose Verbindung der Regelungen zu Forschungserkenntnissen gibt. Vor kurzem sind sogar entgegen aktueller Erkenntnisse zu langen Arbeitszeiten und Risiken (vgl. Fischer et al. 2017; Folkard et al. 2006; Wirtz 2010) der 12-Stunden-Tag und die 60-Stunden-Woche im österreichischen Arbeitszeitrecht verankert worden (durchschnittlich dürfen 48 Wochenstunden innerhalb von 17 Wochen geleistet werden). Zusätzlich entfällt die bisher notwendige arbeitsmedizinische Beurteilung bei Ausweitung der Höchstarbeitszeiten in Betrieben ohne Betriebsrat. Damit werden auch - im Gegensatz etwa zu Risikomanagementsystemen wie in Australien oder des expliziten Auftrags zur Berücksichtigung im deutschen Arbeitszeitgesetz ${ }^{2}$ - Forschungsergebnisse weniger systematisch berücksichtigt.

Zur Überwindung dieser Schwächen und Nachteile schlagen wir einen Ansatz für eine Neue Normalarbeitszeit vor, der eine Bewertung und adäquate Kompensation der physischen und psycho-sozialen Belastung aus Arbeitszeiten ermöglichen und Anreize für einen Einsatz belastender Arbeitszeiten über das versorgungspolitische (zum Beispiel Nachtdienst in Krankenhäusern) und technologisch beziehungsweise wirtschaftlich erforderliche Minimum hinaus senken soll. Die aktuelle arbeitszeitpolitische Diskussion bewegt sich häufig in den Themenbereichen der Flexibilisierung, Individualisierung und Variation der Arbeitszeit im Lebensverlauf - unser Ansatz soll diese um ein weiteres Thema, die adäquate Kompensation belastender Arbeitszeiten, ergänzen.

\section{Der Weg zu einer neuen Normalarbeitszeit}

Als Normalarbeitszeit wird allgemein die Arbeitszeit von Montag bis Freitag zwischen 8 und 17 Uhr bezeichnet (Grzywacz 2017). Als Regel ist diese sozialpolitisch wünschenswerte Arbeitszeit jedoch nicht mehr anzusehen, vielmehr findet sich ein hoher Anteil Beschäftigter in abweichenden Arbeitszeiten wie zum Beispiel Schichtarbeit, Wochenendarbeit, Abendarbeit oder flexiblen Modellen. So arbeiten 43\% der unselbständig Beschäftigten in Deutschland mindestens einmal pro Monat am Wochenende und 20\% in Arbeitszeiten außerhalb von 7 bis 19 Uhr (vgl. BAuA 2016). Die Vielfalt der Arbeitszeit hat insgesamt deutlich zugenommen (Lehndorff 2017).

2 §6 Abs 1 ArbZG (1994): „Die Arbeitszeit der Nacht- und Schichtarbeitnehmer ist nach den gesicherten arbeitswissenschaftlichen Erkenntnissen über die menschengerechte Gestaltung der Arbeit festzulegen.“ 
Von der klassischen Normalarbeitszeit abweichende Arbeitszeiten, wie etwa Arbeit an Abenden, Wochenenden oder in der Nacht sowie unregelmäßige oder lange Arbeitszeiten, sind jedoch mit erhöhten Risiken für die Gesundheit sowie die soziale Teilhabe verbunden. Das erhöhte Unfallrisiko bei langen täglichen Arbeitszeiten ist inzwischen sehr gut belegt (Fischer et al. 2017; Folkard et al. 2006; Wagstaff/Sigstad Lie 2011). Schichtarbeit mit Nachtarbeit verursacht zudem in der Regel eine Desynchronisation der Arbeitszeit mit biologischen und sozialen Rhythmen und hängt daher mit Schlafstörungen, Magen-Darm-Problemen, psychischen Beeinträchtigungen und erhöhten Unfallrisiken ebenso wie mit einer schlechteren Work-Life-Balance zusammen (siehe z.B. Arendt 2010; Arlinghaus/Lott 2018). Aber auch Abend- und Wochenendarbeit sowie variable im Sinne von unregelmäßigen Arbeitszeiten zeigen ähnliche Zusammenhänge (Greubel et al. 2014; Wirtz et al. 2011).

Nicht für alle Beschäftigten ist Arbeit von Montag bis Freitag von 8 bis 17 Uhr jedoch gleich gut oder schlecht - je nach individueller Situation und der Belastung durch die ausgeübte Tätigkeit dürften sich deutliche Unterschiede ergeben. Zudem nimmt die Erreichbarkeit durch mobile Geräte und damit weitere Flexibilisierung der Arbeitszeit und des Arbeitsortes zu, was für manche eine Erleichterung, für viele aber auch eine mögliche zusätzliche Belastung darstellen dürfte (Arlinghaus 2017).

Neben der Veränderung der Arbeitszeiten weg von der klassischen Normalarbeitszeit ist der zunehmende Wunsch auf Seiten der Beschäftigten nach selbstbestimmt flexiblen Arbeitszeiten zu beobachten. Solche Arbeitszeiten sind zum Beispiel durch flexible Startund Endzeiten gekennzeichnet und erlauben im Rahmen der betrieblichen Möglichkeiten eigene Einflussnahme. Etwa 40\% der Beschäftigten in Deutschland haben die Möglichkeit, Einfluss auf Arbeitsstart und -ende zu nehmen, wobei es naturgemäß deutliche Branchenunterschiede gibt (BAuA 2016). Eine solche Autonomie über die Arbeitszeit hat nachweislich positive Wirkungen auf die Gesundheit und das soziale Wohlbefinden der Beschäftigten (vgl. Albertsen et al. 2008; Janßen/Nachreiner 2004). Jedoch kann sie die potenziellen negativen Auswirkungen ungünstiger Arbeitszeiten (z.B. lange Arbeitszeiten, Wochenendarbeit) nicht vollkommen abfedern (Arlinghaus/Nachreiner 2016). Dem steht häufig der Druck der Unternehmen nach mehr bedarfsorientierten und damit aus der Sicht der Beschäftigten fremdbestimmten flexiblen Formen der Arbeitszeitgestaltung gegenüber.

Die klassische Normalarbeitszeit sollte daher aus unserer Sicht modifiziert werden, um den Aspekt der Selbstbestimmung zu integrieren und die festen Zeiten von 8 bis 17 Uhr zu entschärfen. Die Neue Normalarbeitszeit sollte sich daher an einer Gleitzeit von Montag bis Freitag während des Tages (nicht am Abend oder Wochenende) und mit Übergängen und Ausreißern zu anderen Zeiten orientieren. Derartige Ausreißer oder Abweichungen können zum Beispiel maximal 15 Minuten arbeitsbedingte Email-Checks oder Telefonate extra pro Tag sein, unter Berücksichtigung betrieblicher Interessen dies sollte aber ähnlich wie Gleitzeit nicht verpflichtend für die Beschäftigten sein und auch eine zusammenhängende Erholungszeit, wie mit 11 Stunden auf europäischer Ebene festgelegt, sollte dadurch nicht unterschritten werden. 


\section{Belastungsausgleich durch mehr Zeit statt mehr Geld}

Ausgehend von der Neuen Normalarbeitszeit möchten wir einen Ansatz vorstellen, der von mehreren, ineinander greifenden Faktoren ausgeht, mit der Zielsetzung, ein flexibles und für Beschäftigte, Organisationen und Allgemeinheit vorteilhaftes System zu schaffen. Dieser Ansatz zielt auf eine Kompensation der Belastung, die aus bestimmten Arbeitszeitmerkmalen resultiert, durch zusätzliche Freizeit statt durch Geld ab.

Ein solcher Kompensationsansatz ist aus Belastungs- und Beanspruchungssicht angemessen, da durch die Arbeitszeiten eine höhere Belastung und Beanspruchung erfolgt, die durch zusätzliche Freizeit (und damit mehr Erholungszeit, beziehungsweise sozial gut nutzbare Zeiten) wieder abgebaut werden kann. Auch aus sozialer Sicht können belastende Zeiten durch Freizeit an anderen Tagen zumindest teilweise ausgeglichen werden. Finanzielle Zulagen sind in dieser Hinsicht keine angemessene Belastungskompensation.

Bei jüngeren Beschäftigten findet zudem ein Wertewechsel statt, durch den Faktoren wie Work-Life-Balance und das Privatleben zunehmend wichtiger werden. So steigt die Bereitschaft, anstelle finanzieller Zulagen für belastende Arbeitszeiten ein Mehr an Freizeit einzutauschen. Durch eine solche Umwidmung der Zulagen können ergonomisch vorteilhaftere Arbeitszeitsysteme geplant werden. In einem vollkontinuierlichen Schichtsystem kann zum Beispiel statt eines 4-Schicht-Modells mit hoher Wochenarbeitszeit und Nacht- und Wochenendzuschlägen ein 5-Schicht-Modell mit kürzerer Wochenarbeitszeit bei Bezahlung für eine Vollzeitarbeit, aber ohne weitere Zulagen geplant werden. Die in Evaluationen gezeigten Verbesserungen des Krankenstandes sowie des Schlafes und Stressempfindens nach einer Arbeitszeitreduktion (Pfeil et al. 2014; Schiller et al. 2017) stützen diesen Ansatz. Die Komponenten des vorgeschlagenen Kompensationsansatzes werden im Folgenden genauer beschrieben.

\section{Bewertung von Arbeitszeitmodellen im Vergleich zur ,Neuen Normalar- beitszeit'}

Basis der Betrachtung sind jene Belastungen, die sich bei gleicher Tätigkeit und gleichen Umfeldbedingungen (wie etwa Lärm) in der Neuen Normalarbeitszeit ergeben. Andere Arbeitszeitorganisationsformen werden danach bewertet, ob sie - nach jeweiligem wissenschaftlichem Stand - in den Dimensionen Unfallrisiko, Gesundheit, Soziales und Wohlbefinden zumindest gleich gut sind. Wenn nicht, sollten zusätzliche Belastungen in Zeit statt in Geld vergütet werden, um derartige Belastungen durch gezielte Entlastung in Form von mehr Frei-/Erholungszeiten wieder auf das normale Belastungsma $\beta$ zu reduzieren. Die Kernpunkte sind in Abbildung 1 dargestellt. 
Abbildung 1: Ansatz für ein neues System der Regulierung von Arbeitszeiten

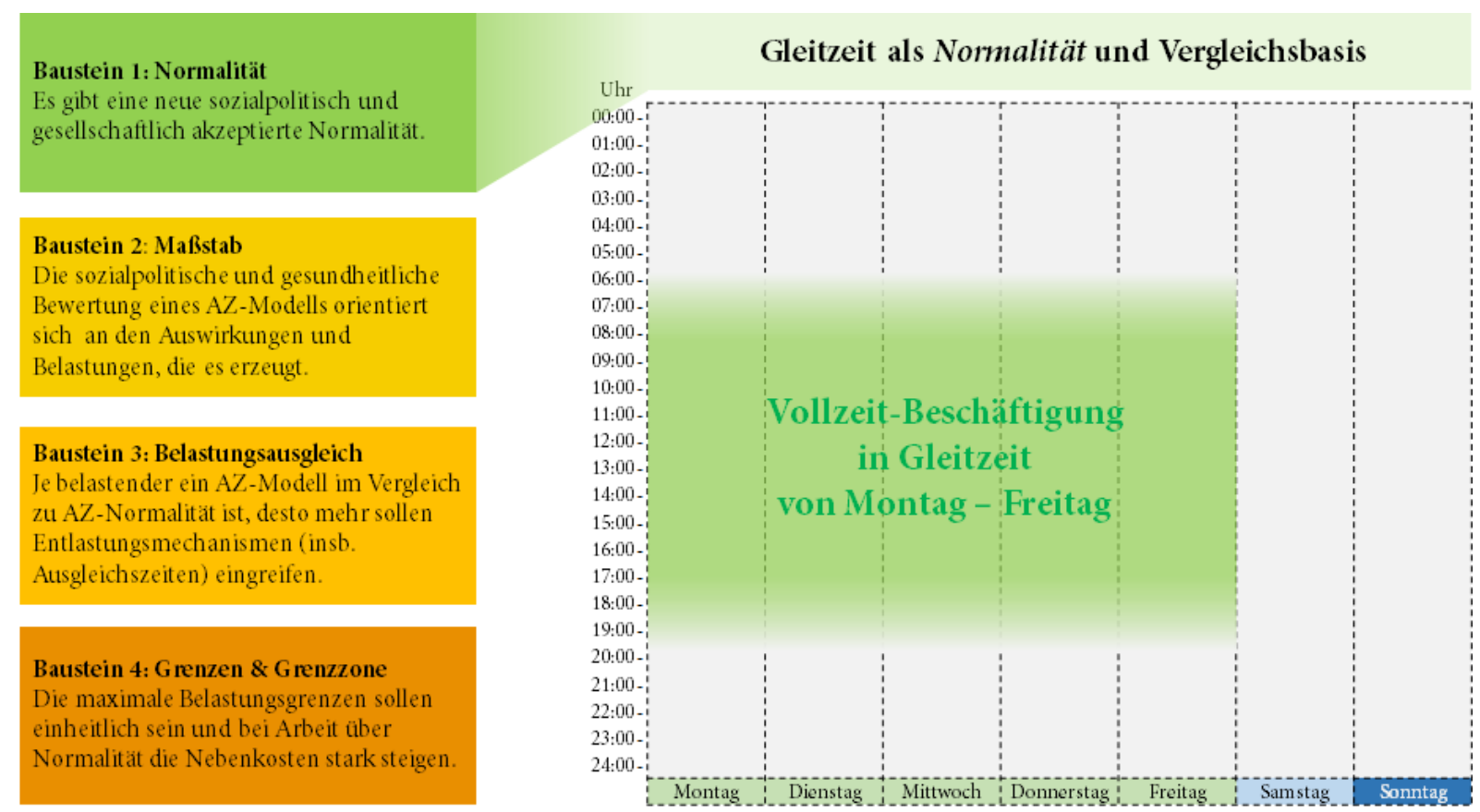

Quelle: eigene Darstellung

\section{Obergrenzen der Belastung und finanzielle Anreize}

Wenn Personen mehr verdienen wollen, sollen (betrieblich nötige) Überstunden möglich sein, aber die zeitlichen Entlastungsfaktoren sollten dadurch nicht konterkariert werden (explizit nicht angedacht ist, dass Personen ihren Zeitausgleich nutzen, um mehr Überstunden leisten zu können) und die Überstunden überwiegend in Zeit abgegolten werden. Der Umfang der resultierenden Arbeitszeit sollte von der Belastung her jenem entsprechen, der für Gleitzeit von Montag bis Freitag (untertags, wie in Abbildung 1) als Obergrenze definiert ist. Damit ergäbe sich ein einheitlicher Belastungsrahmen für alle Beschäftigten.

Um dem Dilemma der finanziellen Anreize für belastende Arbeitszeiten zu entkommen und auch aus staatlicher Sicht ein Lenkungsinstrument im Sinne der Gesundheitsförderung zu etablieren, wird bei steigender Belastung gegenüber dieser Normalarbeit eine progressive Abgabe (zum Beispiel an die Unfall- und Sozialversicherung gekoppelt) angedacht, um auch die höheren Kosten für die Allgemeinheit (zum Beispiel die höhere Unfallgefahr bei Arbeitsverdichtung) abzubilden. Derzeit gibt es in Österreich eine steuerliche Förderung für die ersten Überstunden jeden Monats, für weitere greifen möglicherweise Erleichterungen bei Sozialversicherungsabgaben (zum Beispiel bei Überschreiten der Höchstbemessungsgrundlage bzw. der Beitragsbemessungsgrenzen in Deutschland für höhere Einkommen), obwohl bei extrem hohen Überstundenniveaus und in Folge stressbedingter Erkrankungen (wie Burn-out) die Allgemeinheit höhere Kosten zu tragen hat (DAK 2015; Lohmann-Haislah 2012). 
Wie in Abbildung 2 dargestellt ist, würden so belastende Arbeitszeiten sowohl für Unternehmen als auch für Beschäftigte finanziell weniger attraktiv und die Gestaltung gesunder Arbeitszeiten belohnt werden.

Abbildung 2: Kosten für und Attraktivität von Arbeitszeiten im Zusammenhang mit der Belastung durch die Arbeitszeit

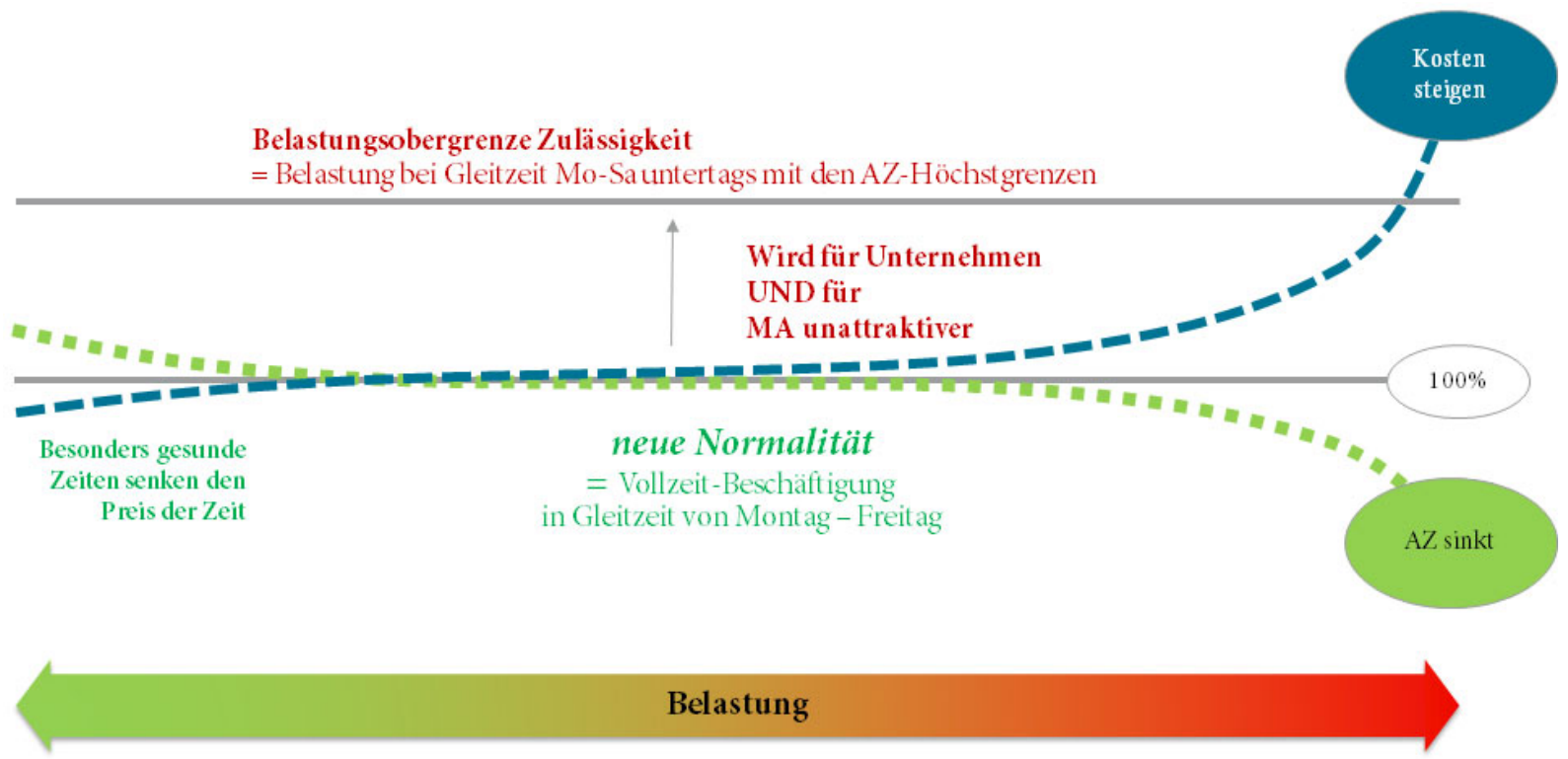

Quelle: eigene Darstellung

\section{Software-Unterstützung für die Berechnung der zeitlichen Kompensation}

Da die Ermittlung der notwendigen Arbeitszeitreduktion zum Ausgleich von Belastung keineswegs trivial ist, wird derzeit eine Softwarelösung zur Unterstützung entwickelt. Dazu wurden in einem Pilotprojekt, dem Time Compensation Calculator 1.0 (vgl. Arlinghaus et al. 2018), verschiedene existierende Risikorechner beziehungsweise Hilfen zur Gefährdungsbeurteilung von Arbeitszeit in eine webbasierte Softwarelösung integriert, um die Risiken spezifischer Arbeitszeitmodelle hinsichtlich Arbeitsunfällen (Spencer et al. 2006), gesundheitlicher Beeinträchtigungen (zunächst Fokus auf Schlafprobleme und Magen-Darm-Probleme, Dittmar et al. 2010) sowie Einschränkungen der sozialen Teilhabe (Hinnenberg et al. 2009) zu berechnen. Eine Benutzeroberfläche erlaubt dabei NutzerInnen, verschiedene Risiko-Szenarien miteinander zu vergleichen (zum Beispiel verschiedene Gewichtungen, verschiedene Definitionen relevanter Zeiten wie etwa für Nachtarbeit).

Im nächsten Schritt werden die ermittelten Risiken mit einer Referenzgruppe verglichen. Diese besteht aus Beschäftigten in Tagarbeit (wobei mittelfristig das Ziel ist, Gleitzeit während des Tages als Referenz heranzuziehen, analog der Neuen Normalarbeitszeit). So wird das relative Risiko des eingegebenen Arbeitszeitmodells detailliert ge- 
schätzt, Tage und Schichtfolgen mit besonders erhöhtem Risiko identifiziert, und die Berechnung einer gegebenenfalls notwendigen Arbeitszeitreduktion ermöglicht, um auf ein vergleichbares Beeinträchtigungs- bzw. Risikoniveau wie die Referenzgruppe zu gelangen. Ein Schema des Tools ist in Abbildung 3 dargestellt.

Eine solche Softwarelösung kann zum Beispiel bei der Aushandlung von adäquaten Kompensationszeiten unterstützen oder auf betrieblicher Ebene Risiken aus der Arbeitszeit bewerten.

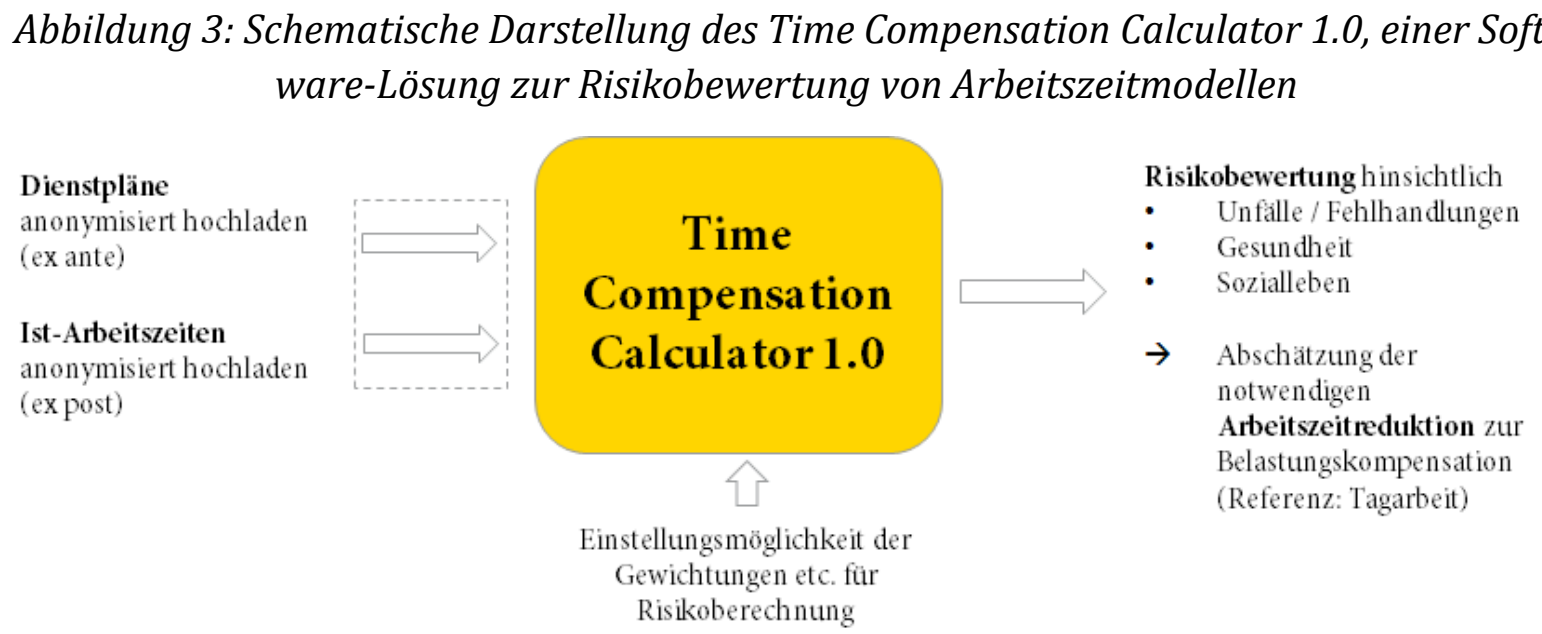

Quelle: eigene Darstellung

\section{Diskussion}

In verschiedenen - jedoch nicht allen - Kollektivverträgen und Tarifverträgen ist bereits eine Freizeitoption enthalten (zu dem österreichischen Kollektivverträgen siehe z.B. Scherz/Schwendinger 2016, zu deutschen Tarifverträgen z.B. Bispinck 2017). Andererseits ist auch mit Widerständen zu rechnen, da ein Einkommensverlust aus Beschäftigtensicht nachteilig wäre. Auch wenn ein Teil der Betroffenen den Gewinn an Zeit zumindest nach einer Gewöhnungsphase höher gewichtet (vgl. Pfeil et al. 2014), ist dies sicher nicht bei allen Beteiligten der Fall. Aus Unternehmenssicht führt eine generelle Arbeitszeitverkürzung zu einem höheren Personalbedarf, wenn mit den kürzeren Arbeitszeiten der gleiche betriebliche Bedarf abgedeckt werden soll. Daher müsste sichergestellt werden, dass eine Arbeitszeitverkürzung bei belastenden Arbeitszeiten nicht zu einer Unterbesetzung führt. Die mögliche Reduktion von Krankenständen und das höhere Wohlbefinden und die Zufriedenheit der Belegschaft wären wiederum als positive Faktoren auch für Unternehmen ins Feld zu führen (eine Diskussion von Teilzeitarbeit findet sich auch bei Scherf und Zander in dieser Ausgabe).

Unser Ansatz adressiert gegenwärtige Schwächen der Arbeitszeitregulierung und versucht diese zu überwinden, ohne die positiven Eigenschaften des jetzigen Systems zu verlieren. Die zentralen Verbesserungen sind die Konzentration auf eine tatsächliche 
Belastungskompensation sowie die Entflechtung von finanziellen Anreizen und von gestaltenden Arbeitszeitfragen.

Die gesetzlichen Rahmenbedingungen zur Gestaltung von Arbeitszeiten sollten dabei an wissenschaftlich fundierten Modellen ausgerichtet werden und Raum für branchenund betriebsspezifische Regelungen ermöglichen. Die Festlegung, wie die Ausgleichsmechanismen zu gestalten wären, würde wiederum von Sozialpartnern in guter Zusammenarbeit mit Wissenschaft und Forschung erarbeitet und in Bezug auf die Bewertungsmodelle von Wissenschaft und Aufsichtsbehörden geprüft werden, um eine Balance von Treffsicherheit und Einfachheit der Regeln zu sichern.

\section{Bibliographie}

Albertsen, Karen, Guðbjörg Linda Rafnsdóttir, Asbjörn Grimsmo, Kristinn Tómasson und Kaisa Kauppinen (2008). Workhours and worklife balance. Scandinavian Journal of Work, Environment \& Health, Suppl. 2008(5), 14-21.

Arbeitszeitgesetz (ArbZG) (1994). Letzte Änderung 17.November 2016.

https://www.gesetze-im-internet.de/arbzg/index.html (Abruf am 15.10.2018).

Arendt, Josephine (2010). Shift work: Coping with the biological clock. Occupational Medicine 60, $10-20$.

Arlinghaus, Anna und Yvonne Lott (2018). Schichtarbeit gesund und sozialverträglich gestalten. Forschungsförderung Report Nr. 3, Düsseldorf: Hans-Böckler-Stiftung. https://www.boeckler.de/pdf/p fofoe report 003 2018.pdf (Abruf am 1.9.2018)

Arlinghaus, Anna (2017). Wissensarbeit - Aktuelle arbeitswissenschaftliche Erkenntnisse. MBFReport Nr. 35, Düsseldorf: Hans Böckler Stiftung.

Arlinghaus, Anna, Johannes Gärtner und Friedhelm Nachreiner (2018). Belastungsausgleich durch Arbeitszeitreduktion - der Time Compensation Calculator 1.0. Tagungsband des 20. Workshop Psychologie der Arbeitssicherheit und Gesundheit 2018, Asanger-Verlag, 717-720.

Arlinghaus, Anna und Friedhelm Nachreiner (2016). Unusual and unsocial? Effects of shift work and other unusual working times on social participation. In: Irena Iskra-Golec, Janet BarnesFarrell und Philip Bohle (Hrsg.) Social and family issues in shift work and non standard working hours. Springer, 39-57.

BAuA (2016). Arbeitszeitreport Deutschland 2016. Dortmund: Bundesanstalt für Arbeitsschutz und Arbeitsmedizin. https://www.baua.de/DE/Angebote/Publikationen/Berichte/F2398.html (Abruf 24.10.2018)

Bispinck, Reinhard (2017). Tarifliche Regelungen zu Arbeitszeiten und Arbeitszeitflexibilisierung in Deutschland. In: Lothar Schröder und Hans-Jürgen Urban (Hrsg.) Gute Arbeit. Streit um Zeit - Arbeitszeit und Gesundheit. Frankfurt a.M.: Bund-Verlag, 92-105.

DAK (2015). Psychoreport 2015. Hamburg: DAK-Gesundheit. https://www.dak.de/dak/download/dak-psychoreport-2015-1718180.pdf (Abruf am 7.6.2018) 
Dittmar, Ole, Friedhelm Nachreiner und Carsten Schomann (2010). Gefährdungsbeurteilung anhand von Merkmalen der Arbeitszeit. Vortrag auf dem GfA-Frühjahrskongress „Neue Arbeitsund Lebenswelten gestalten“. Darmstadt, 24.-26.3.2010. http://www.gawo-ev.de/cms2/uploads/Dittmar\%20GfA\%202010.pdf? phpMyAdmin=8b6ed5803bbabc8d5f96599c9c6997ad (Abruf am 17.04.2018).

Fischer, Dorothee, David A. Lombardi, Simon Folkard, Joanna Willetts und David C. Christiani (2017). Updating the "Risk Index": A systematic review and meta-analysis of occupational injuries and work schedule characteristics. Chronobiology International 34(10), 1423-1438.

Folkard, Simon, David A. Lombardi und Mick B. Spencer (2006). Estimating the circadian rhythm in the risk of occupational injuries and accidents. Chronobiology International 23, 11811192.

Gärtner, Johannes, Peter Döller und Ernst Patka (2014). Das Steuer- und Sozialversicherungsrecht behindert gute Arbeitszeitgestaltung. Zeitschrift für Arbeitswissenschaft 68(2), 116-118.

Greubel, Jana, Anna Arlinghaus und Friedhelm Nachreiner (2014). Arbeitszeitflexibilität auf Kosten der Sicherheit? - Zum Zusammenhang zwischen der Variabilität der Arbeitszeit und der Häufigkeit von Arbeitsunfällen. Zeitschrift für Arbeitswissenschaft 68(2), 89-95.

Grzywacz, Joseph G. (2016). Shift work and its implications for everyday work and family life: A foundation and summary. In: Irena Iskra-Golec, Janet Barnes-Farrell und Philip Bohle (Hrsg.) Social and family issues in shift work and non standard working hours. Wiesbaden: Springer VS, 3-17.

Hinnenberg, Stefanie, Christin Zegger, Friedhelm Nachreiner und Daniela Horn (2009). The utility of time - revisited after 25 years. Shiftwork International Newsletter 24(2), 52. http://www.workingtime.org/resources/Documents/January\%202009\%20Vol.25.1.pdf (Abruf am 17.04.2018)

Janßen, Daniela und Friedhelm Nachreiner (2004). Flexible Arbeitszeiten. Dortmund: Bundesanstalt für Arbeitsschutz und Arbeitsmedizin

Lehndorff, Steffen (2017). Zeit zum Umdenken - Arbeitszeitrealitäten und Arbeitszeitpolitik. In: Lothar Schröder und Hans-Jürgen Urban (Hrsg.) Gute Arbeit. Streit um Zeit - Arbeitszeit und Gesundheit. Frankfurt a.M.: Bund-Verlag, 81-91.

Lohmann-Haislah, Andrea (2012). Stressreport Deutschland 2012 - Psychische Anforderungen, Ressourcen und Befinden.

https://www.baua.de/DE/Angebote/Publikationen/Berichte/Gd68.pdf? blob=publicationF ile (Abruf am 7.6.2018)

Pfeil, Manuel, Daniel Cygan, Johannes Gärtner und Anna Arlinghaus (2014). Auswirkung eines neuen Schichtmodells auf Gesundheit und Mitarbeiterzufriedenheit. Zeitschrift für Arbeitswissenschaft 68, 73-77.

Scherf, Burkhard und Guido Zander (2018). Teilzeit und Lebensarbeitszeit - Bausteine bedarfsorientierter, flexibler Arbeitszeitmodelle. sozialpolitik.ch 2/2018, Artikel 2.3.

Scherz, Eva und Michael Schwendinger (2016). Mehr Zeit statt Geld. Zeitschrift für Arbeitswissenschaft 70(1), 36-39.

Schiller, Helena, Mats Lekander, Kristiina Rajaleid, Carina Hellgren, Torbjörn Åkerstedt, Peter Barck-Holst und Göran Kecklund (2017). The impact of reduced worktime on sleep and per- 
ceived stress - a group randomized intervention study using diary data. Scandinavian Journal of Work, Environment \& Health 43(2), 109-116.

Spencer, Mick, Karen Robertson und Simon Folkard (2006). The development of a fatigue/risk index for shiftworkers. Health and Safety Executive Research Report 446. http://www.hse.gov.uk/research/rrhtm/rr446.htm (Abruf am 17.04.2018)

Wagstaff, Anthony Sverre und Jenny-Anne Sigstad Lie (2011). Shift and night work and long working hours - a systematic review of safety implications. Scandinavian Journal of Work, Environment \& Health 37(3), 173-185.

Wirtz, Anna (2010). Gesundheitliche und soziale Auswirkungen langer Arbeitszeiten. Dortmund/Berlin/Dresden: Bundesanstalt für Arbeitsschutz und Arbeitsmedizin. https://www.baua.de/DE/Angebote/Publikationen/Berichte/Gd59.html (Abruf am 24.10.2018)

Wirtz, Anna, Friedhelm Nachreiner, Katharina Rolfes (2011). Sonntagsarbeit - Auswirkungen auf Sicherheit, Gesundheit und Work-Life-Balance der Beschäftigten. Zeitschrift für Arbeitswissenschaft 65, 134-146. 